\title{
RECENT ADVANCES IN THE DEVELOPMENT OF FERROELECTRIC GENERATORS
}

\author{
L.L. Altgilbers \\ U.S. Space and Missile Defense Command/Army Forces Strategic Command \\ Huntsville, AL 35807
}

\section{Abstract}

Ferroelectric Generators (FEGs) are compact explosive driven high voltage generators. They consist of a small explosive charge, ferroelectric ceramic, and output circuit. There has been and continues to be very active Small Business Innovative Research (SBIR) programs to develop FEGs as very compact power supplies for a variety of loads including antennas, neutron generators, and detonator arrays. In this paper, we will report on some of our recent efforts to improve the performance of FEGs and their components. In addition, we will look at what impact the load (resistive, capacitive, and inductive) has on FEG operation. We will also examine several specific loads that have been powered by FEGs.

\section{INTRODUCTION}

Ferroelectric generators are fairly simple devices in that they consist of a small explosive plane wave charge, one or more ferroelectric ceramic working bodies, and an output circuit. The ferroelectric ceramic is basically an energy storage unit. When electrically poled, they store energy in the form of bound electric charge. When the FEG is shocked, the material is depoled, releasing this stored charge to an output circuit. This depolling is the result of a phase transition that occurs in the ceramic working body. These generators tend to be high voltage sources. By way of example, an FEG with a diameter of $20 \mathrm{~mm}$ and a length of $25 \mathrm{~mm}$ is capable of generating in excess of $100 \mathrm{kV}$.

Lysne $[1,2]$ described two variants of the FEG: axial (or longitudinal) and normal (transverse). In the case of axial FEGs, the shock wave moves along the polling vector, while in the case of normal FEGs, the shock waves moves perpendicular to the polling vector.

The key component of the FEG is the ferroelectric ceramic element. The ceramic typically used in FEGs is lead zirconate titanate (PZT). Of this family of ceramics, most FEG research has been done with either PZT 52/48 or PZT 95/5, where the larger number refers to the mole percentage of zirconium and the smaller number to the mole percentage of titanium in the ceramic. The crystalline structures of PZT can take on different forms depending on the temperature of the material. The crystalline structure determines what phase transitions the ferroelectric will undergo when shocked. For example, PZT 95/5 in the poled state will transition into an antiferroelectric state when shocked.

\section{FERROELECTRIC GENERATORS}

Explosive driven FEGs were first developed by F.W. Neilson at Sandia National Laboratory in 1957. Development continued throughout the 1960s and 1970s, but tapered off in the 1980s and 1990s. Work on FEGs was revived at Texas Tech University in 1999. In 2004, Loki Incorporated, the US Army Space and Missile Defense command (SMDC) along with the US Army Aviation and Missile Research Development Engineering Center (AMRDEC) and the Naval Research Laboratory (NRL) began efforts to develop reliable FEGs that consistently provide high voltages to a variety of loads. Some of their achievements, as well as those of TRS Technologies, Raytheon-Ktech, HEM Technologies, and Radiance Technologies, include:

- Developing reliable transverse FEGs that can generate in excess of $100 \mathrm{kV}$ across capacitive loads using only a few grams of high explosives.

- Demonstrating that the open circuit output voltage of longitudinal FEGs is directly proportional to the percentage of ferroelectric element that is simultaneously compressed.

- Demonstrating that FEGs can drive a variety of loads such as dipole, monopole, and sinuous antennas; vector inversion generators; switches; and microwave sources such as the magnetron.

- Demonstrating higher power, multi-element FEGs.

- Developing an empirical simulated pressure-to-open circuit voltage algorithm for both PZT 52/48 and PZT $95 / 5$, which has demonstrated reasonable correlation between calculated and measured peak voltages over a wide range of pressures [3].

The most recent work in the development of FEGs has been on determining the feasibility of producing multiple electrical pulses. Loki has experimentally shown that

- A compact source with several independent FEG elements in a single body can produce multiple high voltage pulses. The number of pulses generated is equal to the number of PZT elements in the FEG. The inter-pulse time interval can be varied through the variation of the inter-element distance. There were no 
problems with shock wave attenuation and shock wave interference within the epoxy body of multielement FEGs. They did not observe any significant electrical interference between the PZT elements in multi-element FEGs. They found no limitations relative to the further development of this concept and for the creation of multi-element FEGs that are capable of producing a series of ten $150-\mathrm{kV}$ pulses.

- It is possible to generate multiple high voltage pulses with an explosive-driven system based on a series of ferroelectric generators, each activated after a given time delay. Each FEG in the array can contain a large-size single PZT element or several smaller PZT elements. Each FEG in the array operates independently.

- It is possible to generate multiple high voltage pulses with a single-shot system that utilizes a Tesla coil and an explosive-driven FEG as a prime power source. They experimentally demonstrated that PZT 95/5 multi-layer film elements are capable of producing high current in the primary winding of the Tesla coil. The FEG successfully excited the Tesla coil and the coil produced a series of high voltage pulses. It follows from their experimental results that the amplitude of the high voltage pulses depends on the number of turns in the secondary winding of the Tesla coil. Two more parameters also have an effect on the amplitude of the high voltage pulses - one is the amplitude of the current generated by the FEG in the primary winding of the Tesla coil, and the other is the capacitance of the PZT element of the FEG. They found that they could increase the FEG current and PZT element capacitance by an order of magnitude by increasing the number of layers in the PZT element. They found that they can also increase the amplitude of the voltage pulses by increasing the number of turns in the secondary of the Tesla coil, increasing the amplitude of the current generated by the FEG in the primary of the Tesla coil, and increasing the capacitance of the PZT element in the FEG. High-current FEGs could excite a Tesla coil to produce an output voltage of up to $700 \mathrm{kV}$.

- A Tesla coil could not be excited with an explosive-driven high current ferromagnetic generator (FMG). A possible cause of this effect is the absence of capacitance in the FMG circuit; an FMG is a current source of the inductive type with zero capacitance. The Tesla coil is not an air-core transformer. Instead, it is a resonant system that requires capacitance in its primary circuit to operate successfully.

Loki has shown that the advantage of a high-current FEG-Tesla system over a high-voltage FEG by itself is the absence of the high electric field in the explosively shocked part of the device. The high-current FEG (operating via shock wave impact) works at relatively low voltage and low electric field. Their most recent results showed that the FEG operates with $100 \%$ efficiency, i.e. all of the electromagnetic energy stored in the multi-layer PZT 95/5 sample is transferred to the primary winding of the Tesla coil. This means that they have a straightforward way to obtain a series of $1 \mathrm{MV}$ pulses with a single-shot miniature system having a total volume of $100 \mathrm{~cm}^{3}$. An increase in the operating voltage to this level will result in an increase in the microwave radiation to an ultra high power level when a high-current FEG-Tesla system is used as the power source and power conditioning portion of a microwave generator.

Radiance has shown that it is possible for multi-pulse FEG systems to generate pulses at 3 microsecond intervals to 3 millisecond intervals. This was made possible by using a diode array in their output circuit. When the diode array was not used, the first FEG would charge the load plus the other FEGs in the array. Radiances modular array with diode latches was able to charge a $31 \mathrm{nF}, 75 \mathrm{kV}$ capacitor bank to $40 \mathrm{kV}$. The system produced and captured approximately 25 joules of energy. They are currently building a $2 \mathrm{~kJ}$ multipulse system.

Ktech is developing a technique to double the charge per unit of time from a PZT brick.

\section{FERROELECTRIC CERAMICS}

Ferroelectrics are dielectric materials that exhibit electric polarization within specific domains, which can be reoriented within the ceramic working body when an external electric field is applied. Ferroelectric ceramics like PZT can undergo phase transitions as its temperature changes. There is an upper temperature, i.e., the Curie temperature, that varies with the proportions of zirconate and titanate in the ceramic body beyond which the material cannot maintain its poled domain arrangement.

In the case of PZT, poled ceramic working bodies are the result of aligning domains within the crystalline structure. When the PZT is poled, it electrostatically stores energy, imparted by the work done to align the grain boundaries of the individual domains. As pressure is applied to PZT $95 / 5$ in the poled ferroelectric (FE) state, it easily transitions into the unpoled antiferroelectric (AFE) state. Unlike other PZT ceramics, this proximity to the phase boundary ensures that PZT 95/5 undergoes complete depoling.

Shock depolarization measurements have been made. Several different types of PZT were explosively shock depolarized. It is clear that the PZT $95 / 5$ produced by TRS Technologies out performs the other formulations of PZT. While PZT $95 / 5$ is currently the best material for FEGs, work is ongoing to develop ferroelectric materials with enhanced energy storage and enhanced voltage hold 
off. These studies include exploring new dopants, developing new types of ceramics, and developing methods for producing single crystal PZT 95/5. Other ongoing studies include determining optimal FEG designs, matching PZT 95/5 ceramic element sizes to various loads, and determining the impact of the load on FEG operation. The current advantages of PZT 95/5 are:

- High remnant polarization and charge storage density $\left(34-38 \mu \mathrm{C} / \mathrm{cm}^{2}\right)$.

- High internal electrical breakdown strength $(6-7$ $\mathrm{kV} / \mathrm{mm}$ ).

- High charge-discharge efficiency $(80-90 \%)$, since the energy is stored in a field induced phase transition.

- Fast discharge times.

- High energy densities $\left(>10 \mathrm{~J} / \mathrm{cm}^{3}\right)$.

Some recent accomplishments are:

- The identification of multi-layered compositions with remnant poled states around $40 \mu \mathrm{C} / \mathrm{cm}^{2}$.

- The demonstration of single crystal compositions with remnant poled states of around $47 \mu \mathrm{C} / \mathrm{cm}^{2}$.

- Establishment of a low rate initial production line for PZT 95/5 being used by both Government and industrial labs to develop FEGs for multiple applications. TRS can currently produce about 50,000 blocks per year.

The most current work [4] on ferroelectric materials is focused on developing methods to

- Increase the voltage delivered to high impedance loads.

- Increase the energy density of these materials.

- Extend the functionality of FEGs by creating high current components designed to drive low impedance loads.

- Produce a new family of standardized FEG components for specific capacitive, resistive, and inductive loads.

To achieve these objectives, TRS is working with multilayer ceramic ferroelectric materials. To date, they have increased the maximum electric field output to 140 $\mathrm{kV} / \mathrm{cm}$ for multilayer ferroelectric elements and to 80 $\mathrm{kV} / \mathrm{cm}$ for monolithic ceramic elements. They have found that for 2 elements in series, they very nearly double the voltage for two layer elements vs. monolithic elements. Multilayer constructed FEGs survive long dimensional shock transmission, increasing the rise time for inductive loads. This lengthening of the rise time of the FEG discharge may increase matching between the FEG and an inductive load.

\section{LOAD IMPACT ON FEG OPERATION}

It has been experimentally determined that the design of the FEG and the type of load and its parameters impact
FEG operation. Halpin [5] showed that for resistive loads that the current first increases and then decreases as the shock pressure continues to increase in an axial FEG, while Setchell [6] demonstrated that the current reached and maintained a constant value in the normal FEG, provided a threshold pressure is attained.

It has been experimentally demonstrated by several groups that

- The amplitude of the output voltage pulses increases as the thickness of the PZT increases. However, in the case of axial FEGs, it has been shown that the open circuit voltage does not scale linearly with thickness, if the entire disk is not simultaneously compressed.

- $\quad$ FEGs can reliably generate in excess of $100 \mathrm{kV}$ using only a few grams of high explosives in volumes with a diameter as small as $25 \mathrm{~mm}$.

- FEGs can drive a variety of loads such as horn, dipole, monopole, and sinuous antennas; vector inversion generators; and switches.

- The profile of the shock wave significantly affects the amplitude of the output voltage and current pulses produced by both longitudinal and transverse FEGs. Changing the shock wave profile leads to an increase (or decrease) in the amplitude of the output voltage pulses by a factor ranging up to 2.4 times for both types of FEGs with the same PZT elements.

- For FEGs operating with high resistive loads, the amplitude and full width half maximum (FWHM) of the voltage pulse produced by FEGs are highly reproducible and increases as the thickness of the PZT element increases. In addition, increasing PZT thickness increases the energy produced by the FEG, but reduces the specific energy density stored in the PZT.

- For FEGs operating with resistive loads, the amplitude of the output voltage increases noticeably as the resistance increases. However, the amplitude of the current pulse decreases as the resistance increases. The power and energy transferred to the load increases up to a certain load resistance, after which it decreases.

- For FEGs operating with capacitive loads, the amplitude of the voltage pulse decreases as the capacitance increases. However, the electric charge transferred to the load increases as the capacitance increases. The energy transferred to the load increases up to a certain load capacitance, after which it decreases.

- It is difficult to start with the stored charge in a ferroelectric crystal and predict a maximum voltage, since there are three breakdown paths and one serious loss path. The three breakdown paths are through the potting material, along the surface interface between the PZT and the potting material, and through the PZT itself. The serious loss path appears to be due to the deterioration of the series resistance through the 
PZT as the shock propagates through the crystal due to heating and crushing of the material. For example, using the same size and shape of ceramic working body and the same potting agent processed the same way, it was found that 0.5 and $1.0 \mathrm{nF}$ loads caused breakdown at or near peak voltage and that 1.5 and $2.0 \mathrm{nF}$ loads caused breakdown or internal conduction in the PZT well after peak voltage.

- Certain ferroelectric and potting materials and certain ferroelectric element shapes are better than others for yielding high output voltages. As an example, Loki shot single element generators with rectangular shaped EC-64 and PZT 95/5 elements and one with a cylindrically shaped PZT 95/5 element. The latter provided significantly higher voltages. A comparison of the effects of various potting compounds used in FEGs also showed significant differences in their output.

- FEGs have been used by the US Army Aviation and Missile Research, Development and Engineering Center (AMRDEC) to drive commercial blasting cap arrays [6]. Most of the work to date has focused on resistive and capacitive loads, but not inductive loads. AMRDEC has experimentally verified that FEGs can drive highly inductive loads composed of arrays of $16,36,64$, and 128 blasting caps.

- Ktech (now Raytheon-Ktech) conducted extensive studies of the properties of PZT 95/5 and employed them in the construction of power supplies capable of delivering $80-100 \mathrm{kV}$ to capacitive loads and $100-120 \mathrm{kV}$ to resistive loads.

\section{FEG DRIVEN POWER CONDITIONING CIRCUITS}

In order to get the highest voltages possible and in order to get good impedance matching between the FEG and the load, a power conditioning circuit is needed. The most recent work in power condition is the development of compact vector inversion generators and the development of very compact efficient switches.

One of the major losses in pulsed power systems occurs in the closing switches used in the output circuits. Since FEGs are low energy $(1 \mathrm{~J} / \mathrm{cc})$ power supplies, two efforts to develop compact efficient switches were established.

Loki developed solid state discharge switches in which the losses were reduced to 10-15 mJ. Assuming the energy stored in the FEG is $1 \mathrm{~J} / \mathrm{cc}$ and in previous switches that as much as $50 \%$ of the energy was dissipated in the switches, the Loki switches offer a significant improvement.

Radiance also developed an explosive punch through switch and found the losses in the switch to be as low as 8 $-10 \%$ of the energy stored in the FEG.
Radiance also developed a diode network that captures the energy from an erected FEG. This diode network can accept the voltage and current load that is supplied by the FEG. This technique allows them to capture all the energy produced by the FEG by a capacitive load. With a matched load and diode network, Radiance was able to capture $10 \mathrm{~J}$ of energy from a single FEG crystal. This corresponds to an energy density of $1 \mathrm{~J} / \mathrm{cc}$.

\section{FEG DRIVEN ANTENNAS}

It turns out that FEGs are excellent power supplies for driving antennas or other high impedance devices. Three examples will be considered here: 1) FEG driven dipole antenna [7] FEG driven sinuous antenna [8], and 3) Loki FEG driven horn antennas.

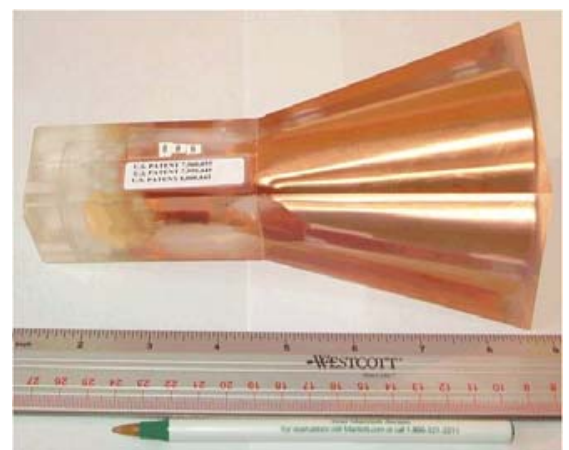

Figure 1. Loki FEG with circular horn (courtesy of J. Baird).

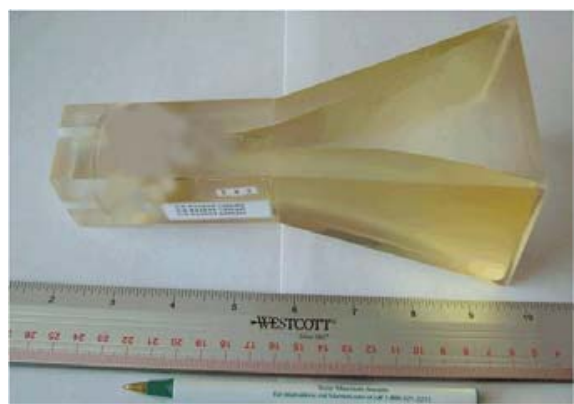

Figure 2. Loki FEG with exponential horn (courtesy of J. Baird).

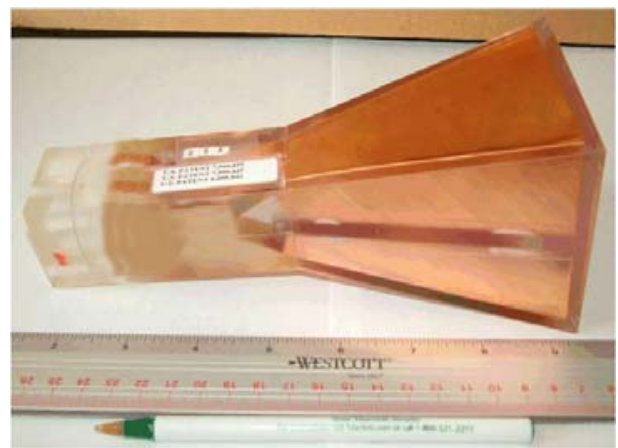

Figure 3. Loki FEG with rectangular horn (courtesy of J. Baird). 
In the case of the dipole antenna, the FEG contained an EC-64 PZT element with the dimensions of $2 \times 1 / 2 \times 1 / 2$ inches and $25 \mathrm{~g}$ of RDX high explosive. The pulse forming network consisted of $280 \mathrm{~mm}$ of RG-58 cable and a spark gap switch. The FEG was capable of generating $40 \mathrm{kV}$ into an open circuit. The dipole antenna was V-shaped, with each arm having a length of $1 \mathrm{~m}$. An identical receive antenna was placed $3 \mathrm{~m}$ from the transmit antenna. Each shot produced what appeared to be two RF bursts that coincided with rapid changes in the source voltage. The first RF burst produced a pulse with peak voltage amplitude of $30 \mathrm{~V}$ peak-to-peak that lasted $1 \mu \mathrm{s}$. The second burst produced a voltage pulse with amplitude of about $50 \mathrm{~V}$ peak-to-peak that also lasted about $1 \mu \mathrm{s}$. Using the peak voltages, the peak power density at the source antenna was estimated to be $1.6 \mathrm{~W} / \mathrm{cm}^{2}$ and the Effective Radiated Power (ERP) was estimated to be $2 \mathrm{MW}$ at the receive antenna, assuming the antennas had a gain of 1 . The frequency of the largest signal was $21.4 \mathrm{MHz}$, which was in good agreement with the predicted value of $21.7 \mathrm{MHz}$.

In the case of the sinuous antenna, the FEG contained two bars of EC-64 with dimensions of $1 / 2 \times 1 / 2 \times 2$ inches and $6.3 \mathrm{~g}$ of explosives. The sinuous antenna consisted of a "hot" side separated from the "ground" side by an insulating dielectric. This separation makes the sinuous antenna suitable for operating at high voltages and adjusting the capacitance of the antenna. The sinuous antenna produces a circularly polarized signal. Note that this single shot system is actually producing a short train of pulses.

Loki developed a series of FEG driven antennas (Figs. 1 -3) that were tested at Redstone Arsenal. The Loki HPM system had a PZT working body with a diameter of 1.5" $(38 \mathrm{~mm})$. The voltage was recorded by a vertical Vivaldi receiving antenna placed on-axis $1 \mathrm{~m}$ from the HPM source.

Radiance has demonstrated that a multi FEG-VIG oscillator operating at only $20 \%$ maximum voltage (Fig. 4) can generate $10 \mathrm{~s}$ of $\mathrm{kV} / \mathrm{m}$ fields at $3 \mathrm{~m}$. The waveform in Fig. 5 was produced by such a system.

\section{FEG DRIVEN MICROWAVE SOURCES}

Ferroelectric generators have been found to be effective compact power sources that produce high voltage pulses driven by an explosive event. However, a difficult problem associated with using ferroelectric generators to power RF devices is that passive device systems such as antennas using basic LC oscillatory waves and spark gaps limit the RF generated by such devices to radiating in the low 100's of MHz band of the spectrum.

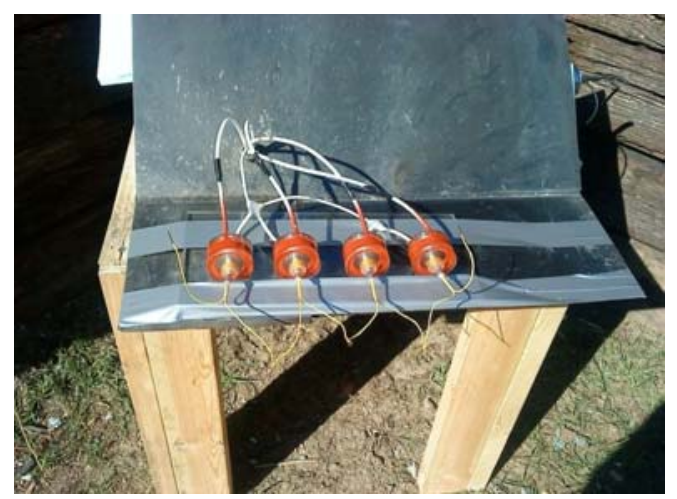

Figure 4. Multi-FEG array built by Radiance (Courtesy of Zachary Roberts, Radiance).

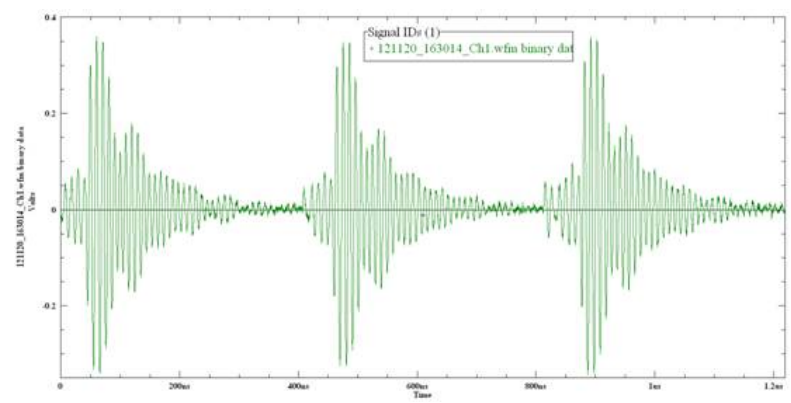

Figure 5. Waveforms produced by a Radiance multi-FEG array driving a VIG (Courtesy of Zachary Roberts, Radiance).

This requires large antennas to efficiently transmit the generated RF pulses and reduces the effective penetration of such RF into targeted electronic systems. In order to operate in the $\mathrm{GHz}$ band, microwave tubes such as magnetrons must be used, but to date none have been successfully coupled to a FEG. In June 2011, HEM Technologies and Texas Tech University [9] successfully coupled a ferroelectric generator to a magnetron. In the initial tests, the ferroelectric material available and the magnetron were greatly mismatched, leading to low efficiency and power output, but characteristics of the system were identified which could greatly improve the overall system design. By rectifying the ferroelectric, the system was also able to produce multiple pulses.

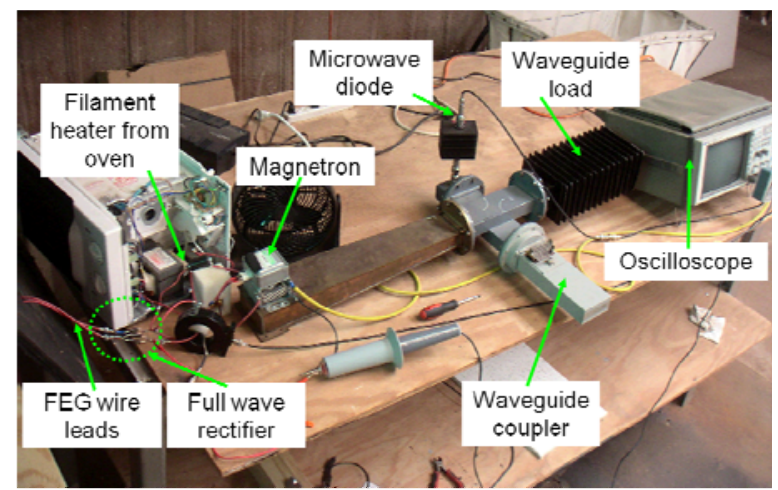

Figure. 6 HEM FEG powering a magnetron (Courtesy of D. Hemmert). 
HEM Technologies and Texas Tech University [9] investigated the feasibility of powering a conventional microwave oven magnetron with a ferroelectric explosive generator (Fig. 6) by coupling a $1.2 \mathrm{~kW}$ magnetron from a $700 \mathrm{~W}$ microwave oven to an FEG through a power conditioning system to match the FEG output to the magnetron. The FEG output was rectified and the voltage was limited by using a resistor. This system delivered two negative high voltage pulses to the magnetron. The ferroelectric material was modified in a second set of experiments to directly drive the magnetron without a power conditioning system.

\section{SUMMARY}

In summary, it has been experimentally demonstrated by several groups that FEGs are reliable single shot high voltage power supplies. They have been successfully used to drive several types of loads.

In order to improve performance of FEGs, it needs to be determined if the voltage limitations of PZT 95/5 are due to enhanced conductivity through the crystal, unexpected shock reinforcement causing premature internal crystal fractures, or partial breakdown at the crystal and potting material interface. Improving performance will also depend on matching the load to the FEG.

In order to improve the performance of FEGs, the impact of their loads on their performance must be better understood. For resistive loads improved performance will come from a high remnant polarization to provide more charge during shock loading. Methods are being investigated to improve their performance by both doping and looking at new materials with higher remnant polarization. The first approach, various doping schemes, should retain the advantages of complete depolarization, but only modestly increase the remnant polarization. The second approach, new ferroelectric materials, has promise for more significant increases in remnant polarization, but shock pressures needed for complete depolarization will likely increase. For capacitive, loads, more benefit can be gained by increasing dielectric breakdown strength rather than from increasing remnant polarization. This allows the generation of higher voltages during discharge and, therefore, higher energy densities for optimally matched loads. The energy density in the load increases as the square of the voltage, but only linearly with charge density. Increases in dielectric breakdown strength are being investigating by a variety of process related issues including density, grain size, chemical stoichiometry, and purity. Issues surrounding inductive loads are not as well characterized, but it is believed that this can be mostly optimized with ceramic element design (e.g. multilayer structure). Such design optimization could also be helpful for capacitive loads by allowing one to better match the voltage generated by the PZT $95 / 5$ with a practical capacitor design.

Distribution A: Approved for Public Release
HEM and TTU has successfully demonstrated that a FEG can produce a high voltage electron beam by means of thermal emission. This allows for the possibility of the FEG being the power source for cheap, compact, single use e-beam sources.

\section{REFERENCES}

[1] Lysne, P.C., 1977: "Resistivity of Shock-WaveCompressed PZT 95/5”, J. Appl. Phys., 48(11), pp. 4565 -4568 .

[2] Lysne, P.C., 1978: "Electrical Response of Relaxing Dielectrics Compressed by Shock Waves: The Axial Mode Problem", J. Appl. Physics, 49 (7), pp. 4180 4185.

[3] Bolyard, D. Neuber, A. Krile, J. and Kristiansen, M., 2010: "Simulation of Compact Explosively Driven Ferroelectric Generators," IEEE Transactions on Plasma Science, 38(4), pp. 1008-1014.

[4] Private Communications from Wes Hackenberger of TRS.

[5] Halpin, W., 1968: "Resistivity Estimates for Some Shocked Ferroelectrics”, J. Appl. Phys., 39 [8] pp. 3821 3826.

[6] Setchell, R.E. Montgomery, S.T. Chhabildas, L.C. and Furnish, M.D., 2000: "The Effects of Shock Stress and Field Strength on Shock-Induced Depoling of Normally Poled PZT 05/5", Shock Compression of Condensed Matter - 1999 (eds. M.D. Furnish et al), AIP Conference Proceedings 505, New York, pp. 979 - 982.

[7] Rader, M.S. Sullivan, C. and Andreadis, T.D., 2005: "Experimental Observation of RF Radiation Generated by an Explosively Driven Voltage Generator", Naval Research Laboratory Report NRL/FR/5745-05-10,122.

[8] Stults, A.H., 2007: "Impulse Loading of Sinuous Antennas by Ferroelectric Generators", IEEE Pulsed Power Conference, Albuquerque.

[9] Hemmert, D.J., Mankowski, J.M., and Altgilbers, L.L., 2011: “A Ferroelectric Explosive Generator Coupled to a Common Microwave Oven Magnetron", $10^{\text {th }}$ Congress on High Current Electronics, Tomsk, Russia.

\section{ACKNOWLEDGEMENTS}

I would like to acknowledgement my team members including Mark Rader at SMDC, Allen Stults at AMRDEC, Jason Baird and Sergey Shkuratov at Loki, David Hemmert at HEM, Frank Rose, Zachary Roberts, and Zachary Shotts at Radiance, Wes Hackenberger and Ed Alberta at TRS, Robert Pap at Accurate Automation, and Bruce Freeman at Ktech. 